\title{
Method Assessment for Non-Targeted Analyses (MANTA) Program: Interlaboratory Study 1 Results
}

Benjamin J. Place

Catherine A. Rimmer

This publication is available free of charge from:

https://doi.org/10.6028/NIST.IR.8384 


\title{
Method Assessment for Non-Targeted Analyses (MANTA) Program: Interlaboratory Study 1 Results
}

\author{
Benjamin J. Place \\ Catherine A. Rimmer \\ Chemical Sciences Division \\ Material Measurement Laboratory
}

This publication is available free of charge from:

https://doi.org/10.6028/NIST.IR.8384

July 2021

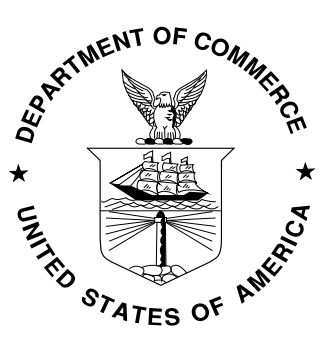

U.S. Department of Commerce

Gina M. Raimondo, Secretary

National Institute of Standards and Technology James K. Olthoff, Performing the Non-Exclusive Functions and Duties of the Under Secretary of Commerce for Standards and Technology \& Director, National Institute of Standards and Technology 
Certain commercial entities, equipment, or materials may be identified in this document in order to describe an experimental procedure or concept adequately. Such identification is not intended to imply recommendation or endorsement by the National Institute of Standards and Technology, nor is it intended to imply that the entities, materials, or equipment are necessarily the best available for the purpose.

National Institute of Standards and Technology Interagency or Internal Report 8384

Natl. Inst. Stand. Technol. Interag. Intern. Rep. 8384, 14 pages (July 2021)

This publication is available free of charge from: https://doi.org/10.6028/NIST.IR.8384 


\begin{abstract}
Non-targeted analysis is the determination of the chemical composition of a complex mixture without a priori knowledge regarding the identities or quantities of the compound. These techniques can be performed in a wide variety of materials and areas of study. Methods and results between laboratories can vary widely based on the compounds of interest and user expertise, which can cause poor interlaboratory comparability of non-targeted results. To address this knowledge gap, three research grade test materials were developed to allow for the comparison of instrument methods, specifically reversed-phase liquid chromatography with positive electrospray ionization mass spectrometry. The materials were shipped to thirtyone participating laboratories to be analyzed by their own non-targeted analysis instrumental method. Of the participants, twenty-three laboratories submitted "good" quality datasets and could be further evaluated. Using principal components analysis, the differences and similarities of the analytical methods could be examined. Overall, there were no obvious trends or associations between the methods of different laboratories and the review of the principal components analysis revealed no notable parameters that caused significant clustering or separation.
\end{abstract}

\title{
Key words
}

non-targeted analysis; environmental analysis; metabolomics; reference materials 


\section{Table of Contents}

1. Background ..................................................................................................................... 1

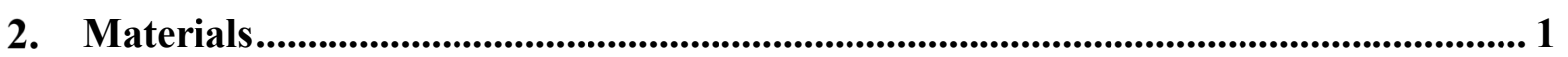

3. Participant Description .................................................................................................... 2

4. Data Analysis Methods.......................................................................................................... 3

5. Results and Discussion ......................................................................................... 4

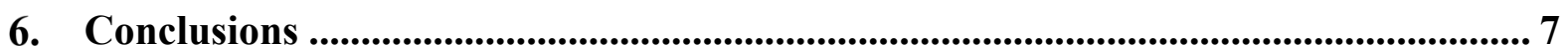

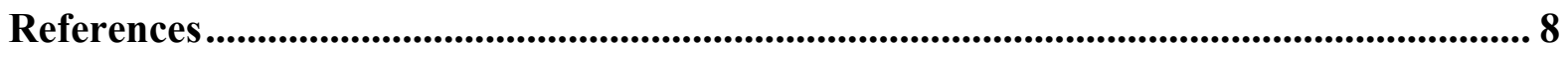

Appendix A: Supplemental Materials.......................................................................................... 9

\section{List of Tables}

Table 1. Chemical composition of the three RGTM solutions, values are mass fractions $(\mathrm{mg} / \mathrm{kg})$ and were determined by gravimetric preparation. ……........................................... 2

Table 2. Participant count of the manufacturers of the liquid chromatographs, mass spectrometers, and chromatography columns used in the study. Values in parentheses are the ratio of the total participants.

Table 3. Participant count of the research areas, participants were able to identify more than one area. Values in parentheses are the ratio of the total participants.

\section{List of Figures}

Figure 1. The results of the quality check for all laboratories (y-axis) for each compound and adduct examined (x-axis).

Figure 2. Principal components analysis scores plot. The labels are the coded laboratory names for the study.

Figure 3. Principal components analysis loadings plot. The labels are the coded figures of merit.

Figure 4. Principal components analysis scores plot. The lines represent the mean score \pm the expanded uncertainty (twice the standard deviation) of laboratories that identified within the environmental (green, dotted), metabolomics (orange, solid) and lipidomics (purple, dashed) communities. 


\section{Background}

Non-targeted analysis (NTA) is a range of techniques aimed at determining the chemical composition of a complex mixture (including food, water, soil, serum, urine, etc.) with no $a$ priori knowledge regarding the identities or quantities of the compounds. This technique can be achieved using a wide variety of instrumentation and methods, which primarily include gas or liquid chromatography with mass spectrometry [1-4]. Reviews and editorial literature have indicated that there is a question of the reproducibility of non-targeted analysis [5-6]. Withinlaboratory variability often can be minimized with a well-defined quality management program, but between-laboratory variability can significantly impact the findings of data produced from different laboratories. This variability can be detrimental to the success of NTA interlaboratory studies.

In response to this need to evaluate the reproducibility of non-targeted analysis methods, researchers at NIST formed the Method Assessment for Non-Targeted Analysis (MANTA) Program, with a focus on measuring the sources of variability across the NTA workflow and determining where reference materials and reference data may reduce the variability and improve interlaboratory comparability of NTA results. For the first study, NIST created a solution of eleven compounds aimed at measuring the instrument response variability. The focus of the first interlaboratory study was the use of liquid chromatography with highresolution mass spectrometry (HRMS).

The solution was ready-to-use for instrumental analysis and shipped to laboratories with different instrumentation and NTA methods. The laboratories were instructed to directly analyze the solution (without sample preparation) and submit the resulting raw analytical data to the MANTA Program coordinators. The method limitations included reversed-phase liquid chromatography with electrospray ionization in positive polarity mode and a $\mathrm{MS}^{1}$ scan range of (at least) $m / z 75$ to 350 .

\section{Materials}

The solution, referred to as Research-Grade Test Material (RGTM) 10104, consisted of a methanol and toluene solution with eleven organic compounds (Table 1). Approximately 1.2 $\mathrm{mL}$ of the solutions were transferred to individual amber glass ampoules that were evacuated with argon. The ampoules were stored at $7^{\circ} \mathrm{C}$ until shipment. 
Table 1. Chemical composition of the RGTM solution, values are mass fractions $(\mathrm{mg} / \mathrm{kg})$ and were determined by gravimetric preparation.

\begin{tabular}{|c|c|}
\hline Compound & $\begin{array}{c}\text { RGTM } \\
10104 \\
\end{array}$ \\
\hline decyltrimethylammonium bromide (DTMA) & 0.39 \\
\hline 18-crown-6 ether (CE) & 0.87 \\
\hline methyl propionate (C3) & 30.95 \\
\hline methyl butyrate (C4) & 19.19 \\
\hline methyl hexanoate (C6) & 9.12 \\
\hline methyl octanoate (C8) & 10.80 \\
\hline methyl decanoate (C10) & 9.83 \\
\hline methyl laurate (C12) & 8.73 \\
\hline methyl myristate (C14) & 20.12 \\
\hline haloperidol (HAL) & 0.88 \\
\hline thiourea $(\mathrm{T})$ & 1.19 \\
\hline
\end{tabular}

Upon registration, participants were shipped three ampoules of RGTM 10104. The samples were shipped overnight in an insulated envelope with blue-ice packs. Participants were instructed to store the solutions at or below $7^{\circ} \mathrm{C}$ until analysis.

\section{Participant Description}

Online registration for the interlaboratory study opened on March $21^{\text {st }}, 2018$, and participants were able to register until August, although sample shipment initially occurred in May $29^{\text {th }}$, 2018. Overall, there were 38 registered participants that received samples. By the close of the study, 31 participants had submitted results.

Upon submission of their results, participants were able to submit instrumental method details and their self-determined research areas. The description of the participants that submitted data is summarized in Tables $\mathbf{2}$ and $\mathbf{3}$. 
Table 2. Participant count of the manufacturers of the liquid chromatographs, mass spectrometers, and chromatography columns used in the study. Values in parentheses are the ratio of the total participants.

\begin{tabular}{|l|c|c|c|}
\cline { 2 - 4 } \multicolumn{1}{c|}{$\begin{array}{c}\text { Liquid } \\
\text { Manufacturer }\end{array}$} & $\begin{array}{c}\text { Mass } \\
\text { Chromatograph }\end{array}$ & $\begin{array}{c}\text { Chromatography } \\
\text { Column }\end{array}$ \\
\hline $\begin{array}{l}\text { ThermoFisher } \\
\text { Scientific }\end{array}$ & $8(25.8 \%)$ & $15(48.4 \%)$ & $1(3.2 \%)$ \\
\hline Agilent Technologies & $12(38.7 \%)$ & $8(25.8 \%)$ & $8(25.8 \%)$ \\
\hline Waters Corporation & $6(19.4 \%)$ & $5(16.1 \%)$ & $14(41.9 \%)$ \\
\hline AB Sciex & & $2(6.5 \%)$ & \\
\hline Bruker & & $1(3.2 \%)$ & \\
\hline Shimadzu & $5(12.9 \%)$ & & $2(3.2 \%)$ \\
\hline GL Sciences & & & $1(3.2 \%)$ \\
\hline Restek & & & $5(16.1 \%)$ \\
\hline Phenomenex & & & \\
\hline
\end{tabular}

Table 3. Participant count of the research areas, participants were able to identify more than one area. Values in parentheses are the ratio of the total participants.

\begin{tabular}{|l|c|}
\cline { 2 - 2 } \multicolumn{1}{c|}{} & $\begin{array}{c}\text { Number of } \\
\text { Raboratories }\end{array}$ \\
\hline Environmental & $10(32.3 \%)$ \\
\hline Exposure Science & $7(22.6 \%)$ \\
\hline Metabolomics & $18(58.1 \%)$ \\
\hline Lipidomics & $11(35.5 \%)$ \\
\hline Nutritional & $1(3.2 \%)$ \\
\hline Industrial/Material Characterization & $0(0 \%)$ \\
\hline Other Clinical/Biochemical Area & $5(16.1 \%)$ \\
\hline Other Research Area & $6(19.4 \%)$ \\
\hline
\end{tabular}

\section{Data Analysis Methods}

After submitting the instrumental method information, participants were invited to submit their raw data to NIST. Prior to submission, the participants were instructed to convert their proprietary data formatted files to mzXML format using ProteoWizard msConvert [7]. The raw data was submitted via the NIST nfiles Secure File Transfer application. All submitted data was available for only 60 days and was deleted from the application after that time.

Data analysis of the raw data files was performed using $\mathrm{R}$ statistical programming language [8] with in-house developed scripts. Briefly, extracted ion chromatograms (EICs) were created for every compound in the solution, based on the exact mass of all compound ions with the participant-reported mass error. These EICs were inspected prior to further analysis for general quality, including the existence of a Gaussian-like chromatographic peak and a good signal- 
to-noise ratio (above 3 times the standard deviation of the noise). After inspection of all EICs, certain quality criteria were determined. The criteria for a "good" quality data file included:

1. At least 3 fatty acid methyl esters were detected at their $[\mathrm{M}+\mathrm{H}]^{+}$ion

2. The $[\mathrm{M}]^{+}$or $[\mathrm{M}+\mathrm{H}]^{+}$ion was detected for decyltrimethylammonium, thiourea, and haloperidol

3. The $[\mathrm{M}+\mathrm{Na}]^{+}$ion was detected for 18 -crown-6 ether

4. All the above compounds formed a detectable chromatographic peak above noise

Once data files were identified as good quality, they were further processed internally by additional R-scripts. Chromatographic and mass spectrometric figures of merit were calculated based on the EICs. Figures of merit are included in the attached data analysis report, but include retention factors, efficiency factor, relative peak area, selectivity factor, signal-to-noise ratio, and adduct formation area ratios. For exploratory statistical analysis, the figures of merit were analyzed using principal components analysis with mean center and autoscale pre-processing and NA values were replaced by the mean value for the specific figure of merit.

\section{Results and Discussion}

There were 31 laboratories that submitted raw data for analysis, of those laboratories only 23 (74 \%) had data that was deemed "good" quality based on the previously discussed criteria. The summary of the quality check for the compounds is visually shown in Figure 1. Laboratories were contacted to re-evaluate the data and only one laboratory re-submitted data, which subsequently passed the quality criteria. Not all laboratories submitted fragmentation $\left(\mathrm{MS}^{2}\right)$ data, therefore only parent ion $\left(\mathrm{MS}^{1}\right)$ data was used for the data analysis.

The most common issues observed were the detection of the fatty acid methyl esters and thiourea; for many laboratories there was no observable chromatographic peak for some of these compounds. More specifically, only two laboratories detected propionic acid methyl ester. The longer-chain fatty acid methyl esters (decanoic acid methyl ester to tetradecanoic acid methyl ester) had the highest frequency of detection of the fatty acid methyl esters. Eight laboratories did not detect thiourea; from a dialogue with some of these laboratories, most did not set their mass range to below $\mathrm{m} / \mathrm{z} 100$ and therefore would not be able to detect thiourea $(\mathrm{M}+\mathrm{H})^{+}$(or propionic acid methyl ester $(\mathrm{M}+\mathrm{H})^{+}$) with a $m / z$ value of 77.0168 .

Using the derived data from the 23 "quality" laboratories, principal components analysis (PCA) was performed. The PCA scores using the first two principal components is shown in Figure 2. The loadings plot for the first two principal components is shown in Figure 3. From the visualization of the first two principal components with loadings, there is no obvious trend or clear association between most laboratories. Of note, laboratories M011 and M013 are from the same laboratory with different users and similar instrument methods and their PCA scores cluster together. The same trend is observed with M012 and M0025, which are from the same laboratory and instrument with different users. These laboratories should yield similar results and the PCA scores support this hypothesis. In contrast, M022 and M027 are two different laboratories and users and do not have significant similarities between instrumental methods.

The three major categories of research for the participants were metabolomics, lipidomics, and environmental analysis. Using the mean and the uncertainty (twice the standard deviation) of 
the first two principal components, regions of the laboratories within these categories are shown in Figure 4. Lipidomics and metabolomics researchers were the same subset of laboratories and their regions are aligned. From Figure 4, there is significant overlap between these three communities, which suggests that there is not a significant method difference between the participating laboratories.

Overall, the solution does appear to discriminate between instrumental methods, although the cause for the discrimination is not clear. The information provided by the PCA is dense and will require further analysis to identify any trends that could impact differences between instrumental methods. All laboratories will be provided with a Microsoft Excel spreadsheet that includes interactive components for laboratories to mine the data for themselves.

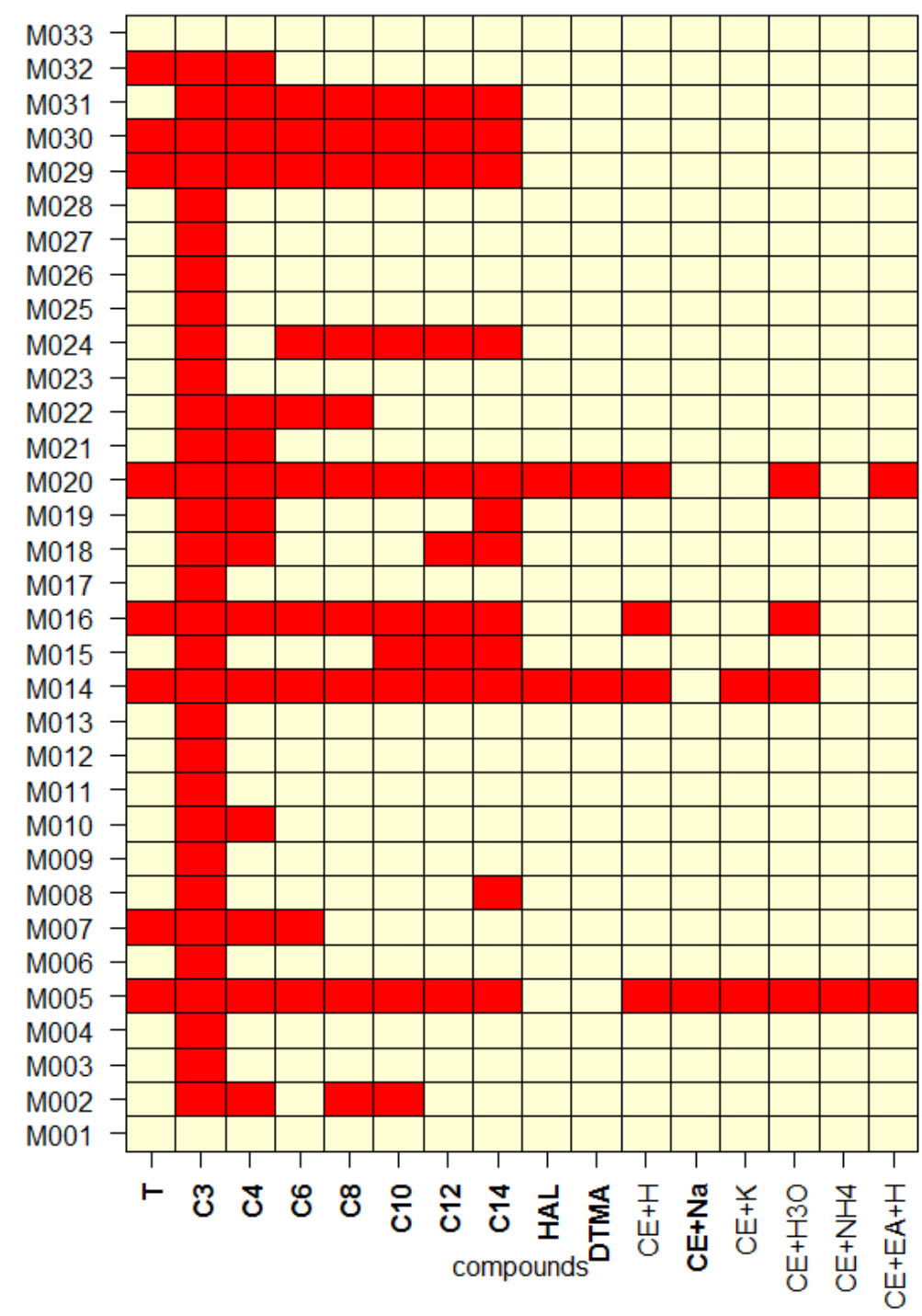

Figure 1. The results of the quality check for all laboratories (y-axis) for each compound and adduct examined (x-axis). Regions that are marked with pale yellow passed the quality check and regions marked with red failed the quality check. Adducts for 18-crown-6 ether are labeled $\mathrm{CE}+\mathrm{H}, \mathrm{CE}+\mathrm{Na}, \mathrm{CE}+\mathrm{K}, \mathrm{CE}+\mathrm{H} 3 \mathrm{O}, \mathrm{CE}+\mathrm{NH} 4, \mathrm{CE}+\mathrm{EA}+\mathrm{H}$ for the respective pseudo 
molecular ions: $[\mathrm{M}+\mathrm{H}]^{+},[\mathrm{M}+\mathrm{Na}]^{+},[\mathrm{M}+\mathrm{K}]^{+},\left[\mathrm{M}+\mathrm{H}_{3} \mathrm{O}\right]^{+},\left[\mathrm{M}+\mathrm{NH}_{4}\right]^{+},[\mathrm{M}+\text { ethylamine }+\mathrm{H}]^{+}$. Compounds in bold were included in the quality check criteria.

\section{Principal Components Analysis of MANTA Interlaboratory Study}

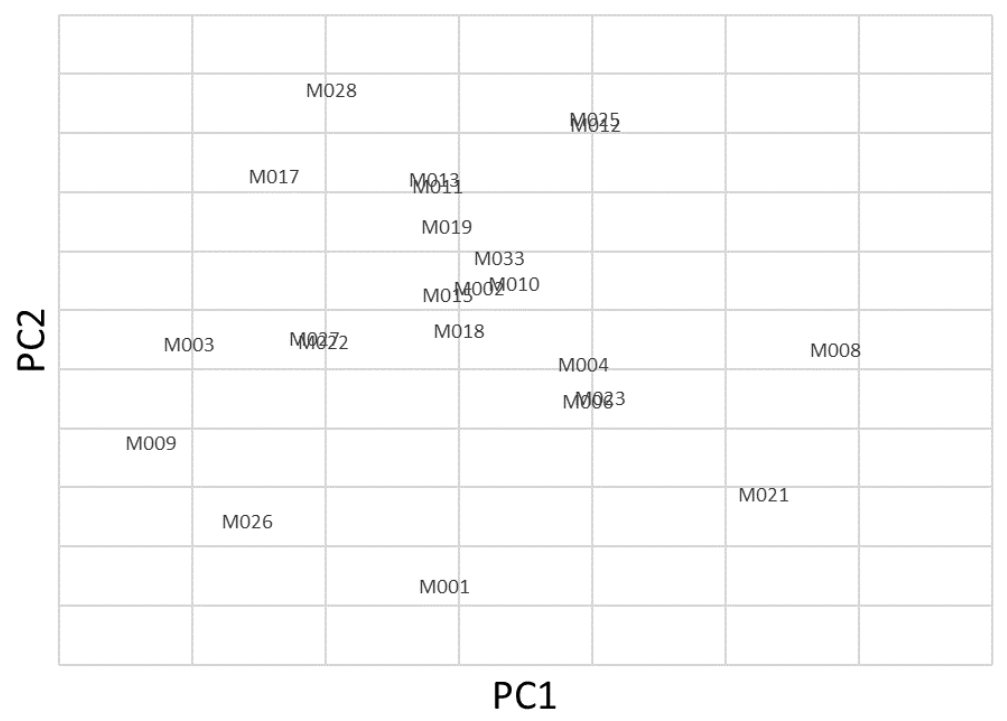

Figure 2. Principal components analysis scores plot using Principal Component 1 (PC1; 27.5 $\%$ variance) and Principal Component 2 (PC2; $13.4 \%$ variance). The labels are the coded laboratory names for the study.

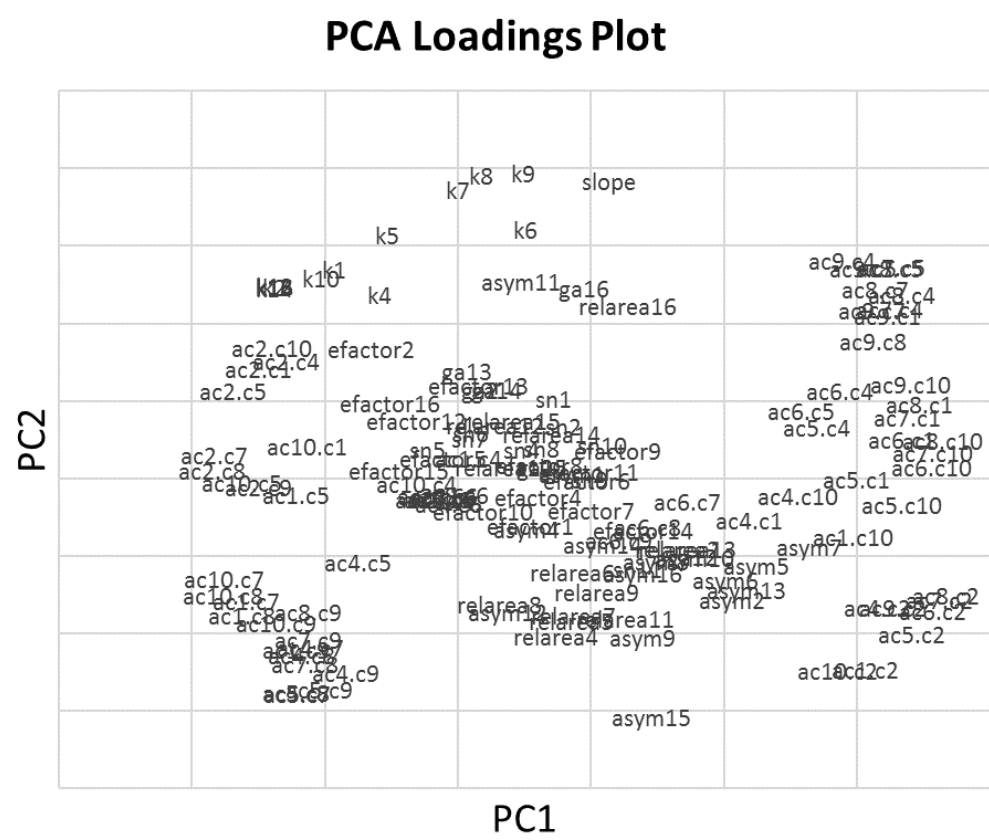


Figure 3. Principal components analysis loadings plot using Principal Component 1 (PC1; $27.5 \%$ variance) and Principal Component 2 (PC2; $13.4 \%$ variance). The labels are the coded figures of merit.

\section{Principal Components Analysis of MANTA Interlaboratory Study}

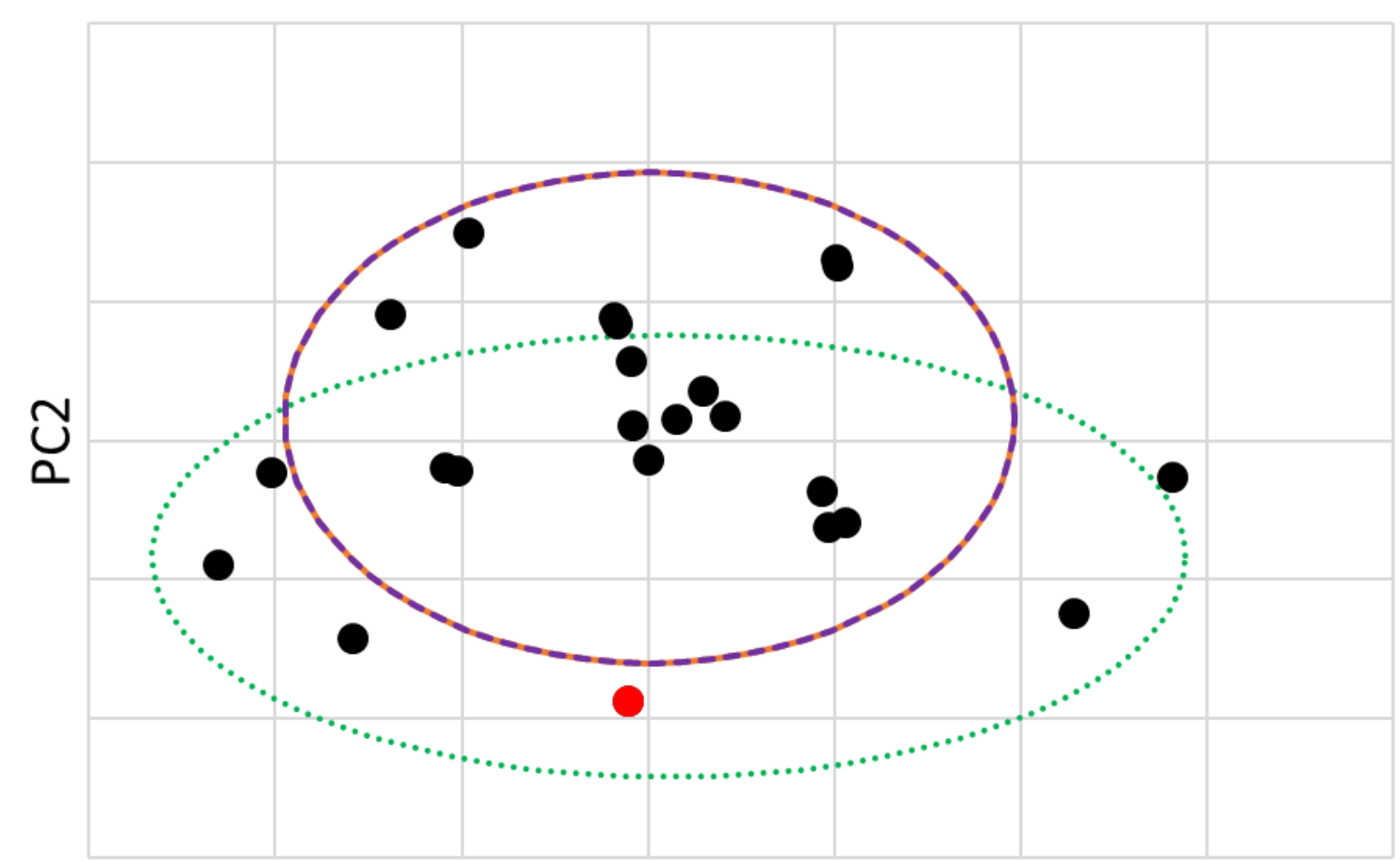

\section{PC1}

Lab Data

Your Data

environmental

metabolomics

lipidomics

Figure 4. Principal components analysis scores plot using Principal Component 1 (PC1; 27.5 $\%$ variance) and Principal Component 2 (PC2; $13.4 \%$ variance). The lines represent the mean score \pm the expanded uncertainty (twice the standard deviation) of laboratories that identified within the environmental (green, dotted), metabolomics (orange, solid) and lipidomics (purple, dashed) communities.

\section{Conclusions}

The results included in this internal report are promising for the use of an artificial solution to determine the similarities and differences between two or more instrumental methods. While there are no clear trends to be drawn from the statistical analysis, there is clustering between laboratories that are using the same instrumentation and analytical method. The data quality check resulted in the removal of many laboratories, suggesting that the solution is not widely usable by laboratories across the disciplines and methods. Future research, with a 
corresponding interlaboratory study, should use the information in this study to develop a new test solution that has a broader applicability.

\section{References}

[1] Andra, SS; Austin, C; Patel, D; Dolios, G; Awawda, M; Arora, M (2017) Trends in the application of high-resolution mass spectrometry for human biomonitoring: An analytical primer for studying the environmental chemical space of the human exposome. Environment International, 100:32-61. https://doi.org/10.1016/j.envint.2016.11.026

[2] Hernandez, F; Ibanez, M; Bade, R; Bijlsma, L; Sancho, JV (2014) Investigation of pharmaceuticals and illicit drugs in waters by liquid chromatography-high resolution mass spectrometry. TrAC Trends in Analytical Chemistry, 63:140-157. https://doi.org/10.1016/j.trac.2014.08.003

[3] Hounoum, BM; Blasco, H; Emond, P; Mavel, S (2016) Liquid chromatography-high resolution mass spectrometry-based cell metabolomics: Experiment design, recommendations, and applications. TrAC Trends in Analytical Chemistry, 75:118128. https://doi.org/10.1016/j.trac.2015.08.003

[4] Ballin, NZ; Laursen, KH (2019) To target or not to target? Definitions and nomenclature for targeted versus non-targeted analytical food authentication. Trends in Food Science \& Technology, 86:537-543. https://doi.org/10.1016/j.tifs.2018.09.025

[5] Hites, R A; Jobst, KJ (2018) Is Nontargeted Screening Reproducible? Environmental Science \& Technology, 52:11975 - 11976. https://doi.org/10.1021/acs.est.8b05671

[6] Metuski, IG; Wakefield, MR; Lynch, K; Stone, J; Fitzgerald, RL (2018) Reproducibility assessment for a broad spectrum drug screening method from urine using liquid chromatography time-of-flight mass spectrometry. Clinical Mass Spectrometry, 8:1 - 7. https://doi.org/10.1016/j.clinms.2018.02.002

[7] Adusumilli, R; Mallick, R. (2017) Data Conversion with ProteoWizard msConvert." Proteomics, 1550:339 - 368. https://doi.org/10.1007/978-1-4939-6747-6_23

[8] R Core Team (2018) R: A language and environment for statistical computing. $R$ Foundation for Statistical Computing, Vienna, Austria. 2018. https://www.Rproject.org/ 


\section{Appendix A: Supplemental Materials}

The source code used for the analysis of the analytical data and the generation of the figures shown in this internal report are available at: https://doi.org/10.18434/mds2-2412 